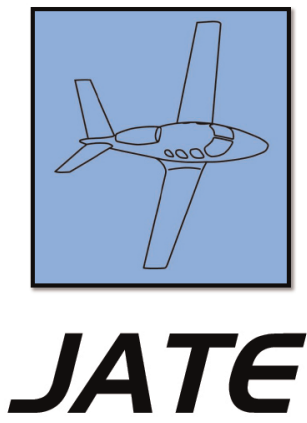

Journal of Aviation Technology and Engineering 2:2 (2013) 56-62

\title{
Integration of Radar System with GPS-Based Traffic Alert and Collision Avoidance System (TCAS) for Approach Control Separation $\dagger$
}

\author{
Ayeni K. Bakare and Sahalu B. Junaidu
}

Ahmadu Bello University

\begin{abstract}
The air traffic control system is a vast network of people and necessary navigational equipment that ensures the safe operation of commercial and private aircraft throughout the world. Air traffic controller service is responsible for area, approach and aerodrome control. Aircraft fatal accident data for commercial jet airplanes between 1959 and 2008 show that 36\% of the accidents happen in the final approach and landing phase, possibly due to mechanical failure or human error resulting from poor communication between the pilot and air traffic controller. This paper presents an integration of a radar system with a GPS-based Traffic Alert and Collision Avoidance System (TCAS) for approach control separation between all instrument flight rules (IFR) flights and between IFR flights and visual flight rules (VFR) flights operated as controlled flights. The integration is simulated using MATLAB. Results of the simulation show that aircraft separation is easier and safer than relying only on an air traffic controller. We note, however, that the service of air traffic controllers is still required for successful aerodrome control.
\end{abstract}

Keywords: radar system, TCAS, IFR, VFR, approach control separation

\section{Introduction}

The air traffic control system is a vast network of people and necessary navigational equipment that ensures the safe operation of commercial and private aircraft throughout the world. The International Civil Aviation Authority (ICAO) in 1965 mandated that air traffic controllers be responsible for area control, approach control and aerodrome control services.

\section{About the Authors}

Ayeni K. Bakare received his BSc (Computer Science with Economics) from Obafemi Awolowo University, Ile-Ife, Nigeria and MSc (Computer Science) from Ahmadu Bello University, Zaria, Nigeria. He is currently a PhD student in the same university with research interest in air navigation aids. Correspondence concerning this article should be sent to bakarre@yahoo.com.

Sahalu B. Junaidu is a professor of computer science in Ahmadu Bello University, Zaria. He received his BSc (Mathematics with Computer Science) from Ahmadu Bello University, Zaria, Nigeria, MSc (Formal Methods in Software Engineering) from Queen Mary and Westfield College, University of London and $\mathrm{PhD}$ (Computer Science) from St. Andrews University, Scotland.

$\dagger$ This work would not have been possible without the advice and support of Mr. Taiwo, a pilot in the Nigerian College of Aviation Technology, Zaria, Nigeria and the staff of the mathematics department at Ahmadu Bello Univerity. We are also grateful to the entire staff of MicroScale Embedded, Ltd., Kaduna, Nigeria for granting use of their laboratory. 
Area control is responsible for maintaining separation between all instrument flight rules (IFR) flights. In order to be operated on an IFR flight plan and fly under IFR, aircraft must be equipped with suitable instrumentation and with navigation equipment appropriate to the route to be flown. Approach control is responsible for providing separation between all IFR flights, and between IFR flights and visual flight rules (VFR) flights for which ATC control has accepted responsibility in the vicinity of an airport. Arriving aircraft are followed by approach control once they have been released by area control until they have landed and cleared the runaway. When departing, aircraft stay with approach control from the time they enter the runway until they have been released to area control. The aerodrome control gives advice and instructions to aircraft taxiing, landing, taking off and flying within the aerodrome traffic zone in order to achieve an orderly and expeditious flow of air traffic, and to assist in preventing collisions between aircraft and either other aircraft or obstructions on the ground. Aerodrome control also assumes control of aircraft, vehicles and personnel within the maneuvering area.

An analysis of fatal aircraft accidents in commercial jet airplanes occurring between 1959 and 2008 showed that $36 \%$ of accidents happened in the final approach and landing phases of flight. Of on-board fatalities, $13 \%$ occurred in the final approach phase and $12 \%$ during landing, for a total of $25 \%$ occurring in a crucial final phase of flight. For a graphical representation of this data, refer to Figure 1, (Kebabjian, 2012). These accidents may have been caused as a result of mechanical failure, human error which resulted from poor communication between the pilot and air traffic controller, or an error in the navigational equipment.

This paper presents the integration of a radar system with a GPS-based traffic alert and collision avoidance system (TCAS) for approach control separation between all IFR flights, and between IFR and VFR flights operated as either arriving or departing controlled flights, in order to reduce aircraft accidents.

In order to better model the effects of integration, the authors integrated a radar system with GPS-based TCAS for approach control separation using MATLAB. Results of the simulation show that aircraft separation is easier and safer than relying only on visual input from an air traffic controller. We note, however, that the service of air traffic controller is still required for successful flight operation, especially for aerodrome control.

Related work will be discussed, followed by an introduction and explanation of the use of MATLAB, radar, TCAS, and automatic dependent surveillance (ADS), along with the requirements and functionality of the simulation. Future research opportunities will be presented, as well.

\section{Related Work}

Air traffic control systems began with the automation of simulator machines as a training tool for both trainees and instructors. One of the earlier simulators is the Tower Research Simulator (TRS); another that is widely used in air traffic control simulation is the Total Airspace and Airport Modeler (TAAM) (Sood \& Wieland, 2003). TAAM was developed by Preston Aviation Solutions in Australia. However, Plaettner-Hochwarth, Zhao, \& Robinson (2000) reported that one of the major drawbacks of this software was the price tag. In 1997, a single site license cost about $\$ 350,000$. In addition to its high price, TAAM was also reported to lack stochastic options and coverage of all Air Traffic Management (ATM) components. Dennis (2003) conducted research using the Tactical Separation Assisted Flight Environment (TSAFE), developed by Heinz Erzberger, chief scientist for air traffic control at the NASA Ames Research Center. TSAFE was developed

\section{Accidents and Fatalities by Phase of Flight}

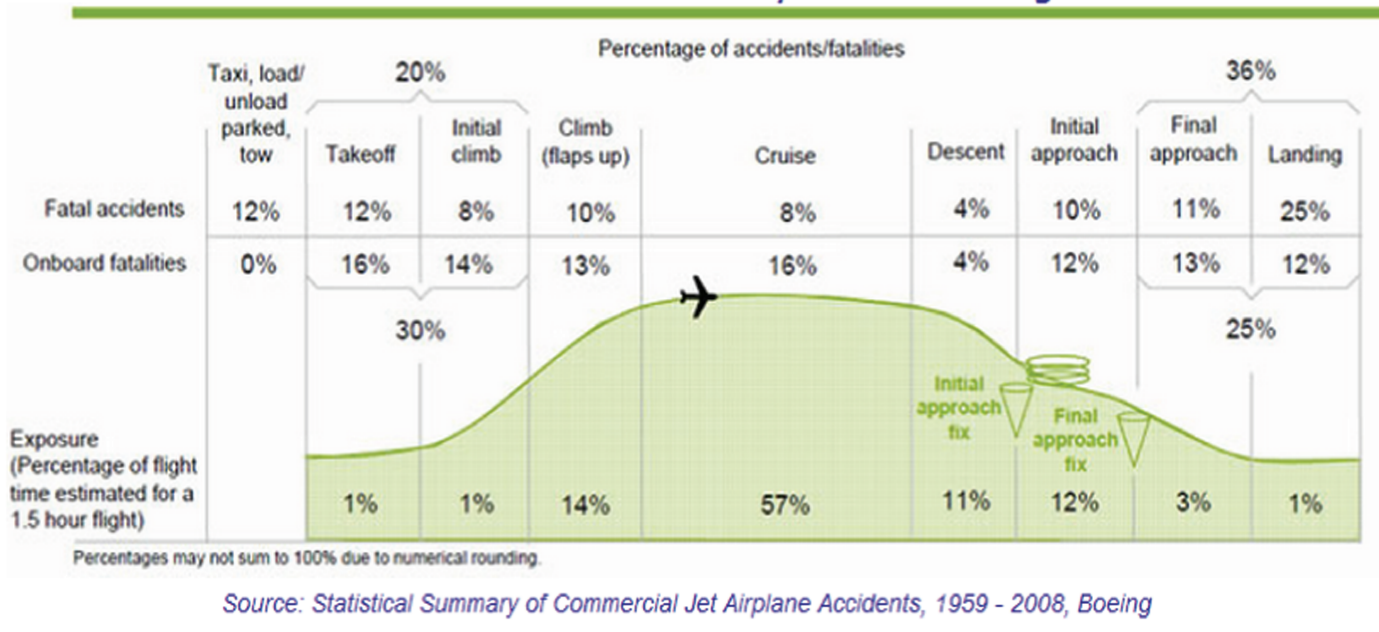

Figure 1. Accidents and fatalities by phase of flight. 
experimentally, with the goal of emulating controller inference, typically consisting of complex heuristics and intricate algorithms.

\section{System Description and Functionality}

There are many navigational aids which provide direction or range for aircraft, including: non-directional beacons (NDB), very high omni-directional range/instrument landing systems (VOR/ILS), and radio detection and ranging (radar) (Obiniyi \& Bakare, 2009). For this research project, a radar system was integrated with GPS-based TCAS for approach control separation. In the following sections, the major components of the system will be briefly discussed.

\section{Radar}

Radar is one of many electronic navigation aids available, but it is unique in that it provides the air traffic controller with a comprehensive view of air traffic over a wide area, and hence has assumed a crucial role in modern Air Traffic Control (ATC) procedures, especially in busy airspaces and particularly under positive control. ATC radar is capable of carrying out the functions and roles of approach control and terminal area control, with the added capability of extended range using secondary surveillance radar (SSR) techniques (International Civil Aviation Organization, 2002). Radar systems' capabilities include use in the provision of air traffic services which have a very high level of reliability, availability and integrity with back-up facilities.

A radar system consists of a number of integrated elements, including radar sensor(s), radar data, transmission lines, radar data processing systems, and radar displays. Multi-radar systems, that is, systems utilizing more than one radar sensor, have the capability to receive, process and display, in an integrated manner, data from all the connector sensors.

Radar is capable of integration with other automated systems used in the provision of air traffic services (ATS), and provides an appropriate level of automation with the three objectives of improving the accuracy and timeliness of data displayed to the controller, reducing controller workload, and minimizing the need for verbal coordination between adjacent control positions and ATC units. GPSbased TCAS can also be integrated with radar systems.

Radar systems are capable of displaying safety-related alerts and warnings, including conflict alerts, minimum safe altitude warning, conflict prediction and unintentionally duplicated SSR codes. Primary surveillance radar (PSR) and SSR may be used either alone or in combination by ATS in order to ensure adequate separation between aircraft, provided reliable coverage exists in the area and the probability of detection, determined by the accuracy and the integrity of the radar system, is satisfactory.
Radar derived data that are available for display to the controller include, as a minimum, radar position indications, radar map information, and SSR Mode A, Mode C, and Mode $\mathrm{S}$ (when available). The radar systems also provide a continuously updated presentation of radar derived information and radar position indications. Navigation guidance to aircraft in the form of specific headings (known as vectoring) is provided by radar. When vectoring an IFR flight, the radar controller issues clearances such that the prescribed obstacle clearance will exist at all times until the aircraft reaches the point where pilot will resume his or her own navigation (Wolff, 2012).

\section{TCAS and Automatic Dependent Surveillance (ADS)}

The traffic alert and collision avoidance system (TCAS) is an aircraft collision avoidance system designed to reduce the incidence of midair collisions between aircraft. Independent of air traffic control, it monitors the airspace around an aircraft for other aircraft equipped with a corresponding active transponder, and warns pilots of the presence of other aircraft which may present a midair collision (MAC) threat. It is mandated by ICAO to be added to those aircraft having MTOM (maximum take-off mass) over 5,700 kg (12,586 $\mathrm{lbs}$ ) or authorized to carry more than 19 passengers. TCASequipped aircraft "interrogate" all other aircraft within a predetermined range of their position (at a carrier frequency of $1030 \mathrm{MHz}$ ) and those aircraft reply (at $1090 \mathrm{MHz}$ ). This interrogation-and-response cycle may occur several times per second. Through this constant back-and-forth communication, the TCAS system builds a three dimensional map of aircraft in the airspace, incorporating their bearing, altitude and range. Then, by extrapolating current range and altitude difference to anticipate future values, it determines whether a potential collision threat exists. If required, it automatically negotiates a mutual avoidance maneuver between the two or more conflicting aircraft. Current TCAS avoidance maneuvers are restricted to the vertical plane only; that is, restricted to changes in altitude and modification of climb/sink rates (Lufthansa Airlines, 2005). There may be instances where the lateral resolution maneuvers suggested in this paper may be incorporated in certain complex traffic conflict scenarios, such as an approach control area between aircraft taking off or landing.

The first generation of collision avoidance technology is TCAS I. It is less expensive but also less capable than the modern TCAS II system. GPS-based TCAS (Bakare \& Sahalu, 2011), a technical upgrade to the TCAS II system which is used in the proposed system, allows reverse logic and increased total separation between aircraft both in lateral and vertical aspects.

Automatic dependent surveillance (ADS) is a system used in the air traffic system in which aircraft automatically transmit, via a data link, data derived from on-board navigation systems. ADS is an air/ground data link 
application. At a minimum, the data includes aircraft identification and a three-dimensional position, but additional data may be provided as appropriate. In order to improve accuracy and timeliness of the control of aircraft on approach, GPS-based TCAS is synchronised with ADS to provide optimum navigation and performance accuracy and automatic data transmission without the need for wide separation standards. The accuracy of approach control separation is expected to be improved with the use of GPSbased TCAS interfaced with ADS, by enabling precise locating through the use of the Global Navigation Satellite System (GNSS). This will provide an easy means of monitoring and determining the position of aircraft.

Aircraft position information is sent in an ADS report to ATC at a rate of at least once every 10 seconds. The report consists of six basic pieces of information-latitude, longitude, altitude, speed, time and accuracy. This is managed, organized, and formatted by the onboard flight management system (Airbus, n.d.)

An ADS reporting agreement specifies what data schedule the aircraft should use and when the report should be sent. There are three types:

1. Periodic contracts: data is transferred or transmitted at fixed repetitive rate.

2. Event contract: data is transmitted each time an event specified by the control system occurs; these include passing away point, speed change, route change, and altitude change, among others.

3. Demand contract: data is to be sent each time a request is made by the control system.

\section{GPS-based TCAS, ADS and the NextGen system}

The concept underlying NextGen is the transformation of Air Traffic Control (ATC) from a ground-based radar network to an aircraft and satellite-based system. Automatic Dependent Surveillance-Broadcast (ADS-B) is the NextGen system (Duncan Aviation, 2013). ADS-Bequipped aircraft will send their identification along with speed and precise vertical and horizontal positions to the Global Navigation Satellite System (GNSS) constellation. This information is instantly broadcast over the entire GNSS network to other ADS-B equipped aircraft as well as ADS-B ground stations at $1090 \mathrm{MHz}$. These stations then pass the information along to ATC in real time.

GPS-based TCAS equipment which is capable of processing ADS-B messages may use this information to enhance the performance of TCAS, using techniques known as "hybrid surveillance. This capability may be incorporated into an upgrade of TCAS.

In the NextGen system, each aircraft has the capability of obtaining real-time data about other aircraft in its vicinity, significantly enhancing safety and efficiency, and directly benefits pilots, controllers, airports, airlines, and the public.

\section{Introduction to MATLAB}

MATLAB is a high-performance language for technical computing. It integrates computation, visualization, and the programming environment (David, 2005). MATLAB is a modern programming language environment with sophisticated data structures; it contains built-in editing and debugging tools and supports object-oriented programming. These factors make MATLAB an excellent tool for teaching and research.

MATLAB has many advantages compared to conventional computer languages like $\mathrm{C}$ or FORTRAN for solving technical problems. MATLAB is an interactive system whose basic data element is an array that does not require dimensioning. The software package has been commercially available since 1984 and is now considered as a standard tool at most universities and industries worldwide. It has powerful built-in routines that enable a very wide variety of computations. It also has easy-to-use graphics commands that make the visualization of results immediately available.

Specific applications are collected in packages referred to as toolboxes. There are toolboxes for signal processing, symbolic computation, control theory, simulation, optimization, and several other fields of applied science and engineering. Version 7.9.0.529 (R2009B) was used for this research.

\section{System Design}

In the approach area, there are typically many aircraft; some might be taking off while others are approaching to land. At an extremely busy airport, it may be difficult for the air traffic controller to maintain the minimum required separation between all aircraft continuously. In order to alleviate some of the resulting controller workload, the proposed system utilizes GPS-based TCAS interfaced with ADS and integrated with radar (Bakare \& Sahalu, 2011). See Figure 2 for a graphical representation.

It is assumed that radar coverage is total; that is, various installations in a particular country are networked so system coverage is complete, taking into consideration radar capability of detecting aircraft within a range of 250 NM. Aircraft within range are detected and identified, and aircraft under surveillance are continuously tracked. Radar systems display their safety-related alerts and warnings, including conflict alerts, minimum safe altitude warning, and conflict prediction to air traffic controllers.

The surveillance and tracking capabilities of radar share trajectory data with that of TCAS, and pilots are able to utilize this information to avoid potential midair collisions. GPS-based TCAS interfaced with ADS can allow for the identification and three-dimensional position of the aircraft. Aircraft equipped with TCAS receive squitter messages transmitted by the transponder of the intruder aircraft 


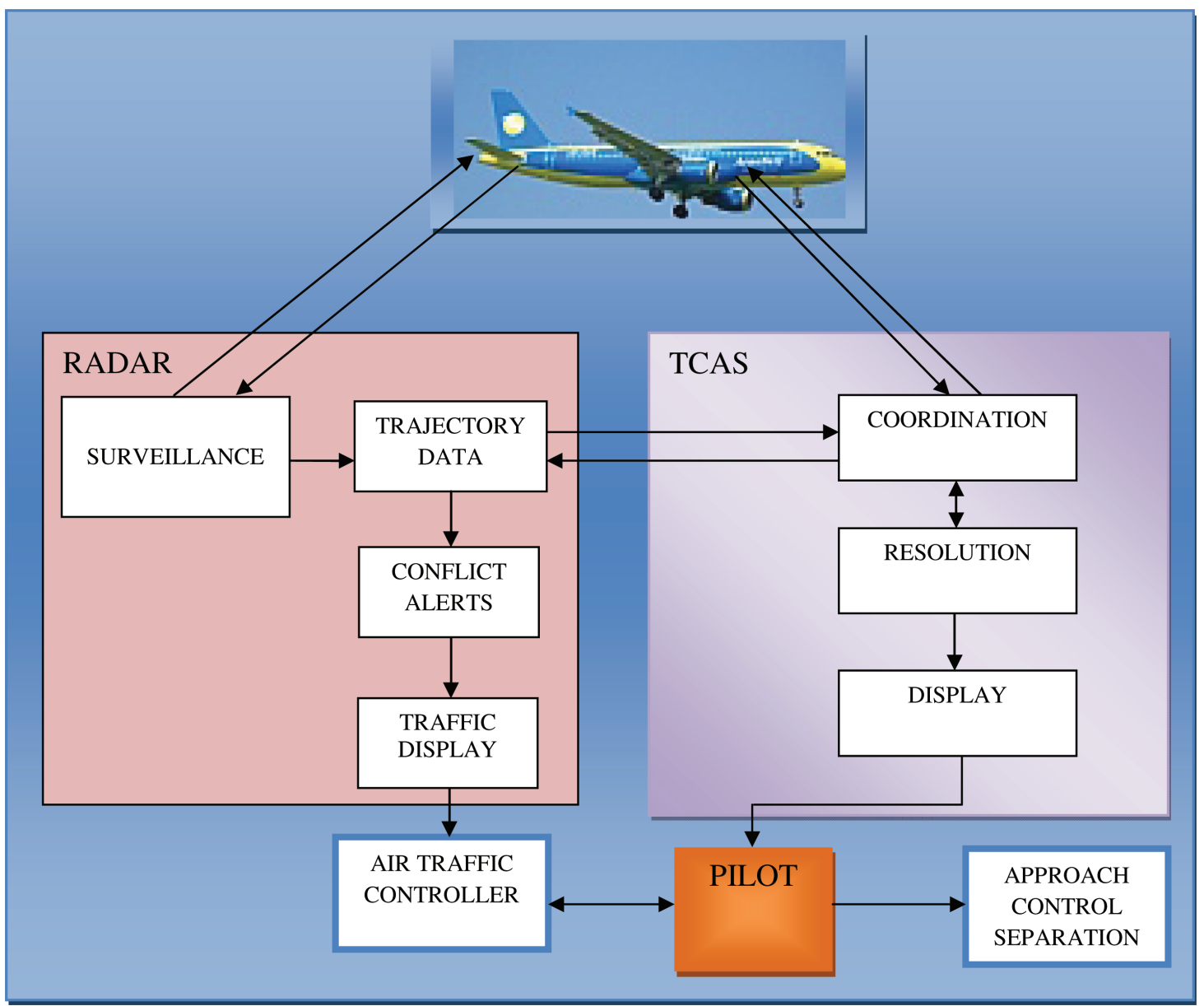

Figure 2. Approach control separation system flow.

(within a range of 14 or $20 \mathrm{NM}$ ). The transmission occurs periodically at one second intervals, and intends to enable its detection and identification. Through repetitive TCAS interrogations, up to a combined total of 30 intruders can be tracked at any one time.

In an aircraft equipped with transponders, TCAS is active and transmits pulses. Aircraft under surveillance are tracked continuously, with a nominal time interval of one second between interrogations. If another aircraft comes within a specified distance, decisions about avoidance maneuvers are determined and issued appropriately. A coordination procedure is initiated between the two aircraft, after taking into consideration the vertical status (altitude) of the aircraft, its sensitivity level, and maximum speed. TCAS issues preventive traffic advisory (TA) or horizontal corrective resolution advisories (RA), as the case may be. This system is simulated using MATLAB as shown in Figure 3.

\section{Aircraft Simulation in Different Altitude Modes for Approach Control Separation}

In the approach control area, many different operations are conducted simultaneously. Some aircraft are taking off and changing altitude rapidly, while those in the landing queue are separated vertically by at least 1,000 feet. GPS-based TCAS ensures strict compliance to reduced vertical separation minimums (RVSM) of $1,000 \mathrm{ft}$ to allow for adequate aircraft separation. For instance, lateral resolution maneuvers could be issued to Aircraft B (while approaching to land) and Aircraft A (taking off). Consider the following scenario: Aircraft A takes off from a destination with longitude 7.545750 and latitude 10.769100 , with a cruising speed of $240 \mathrm{~km} / \mathrm{hr}$ selected. Meanwhile, Aircraft B is on approach, with longitude 7.605770 and latitude 10.889200 (see Fig. 3). It can be assumed that Aircraft B is going to land approximately where Aircraft A took off, and that the two aircraft are at different altitudes. Because of the continuous availability of current trajectory data received from GPS, as shown in the server console in Figure 3, corrective lateral resolution advisories can be issued for both aircraft.

Because Aircraft B (descending) cannot instantaneously gain full power to climb and Aircraft A (ascending) cannot instantaneously climb higher or descend, TCAS issues a corrective lateral RA (within 34 seconds) when the intruder range is considered dangerous at the closet point of approach (CPA), against a standard of 30 seconds (see Table 1). 


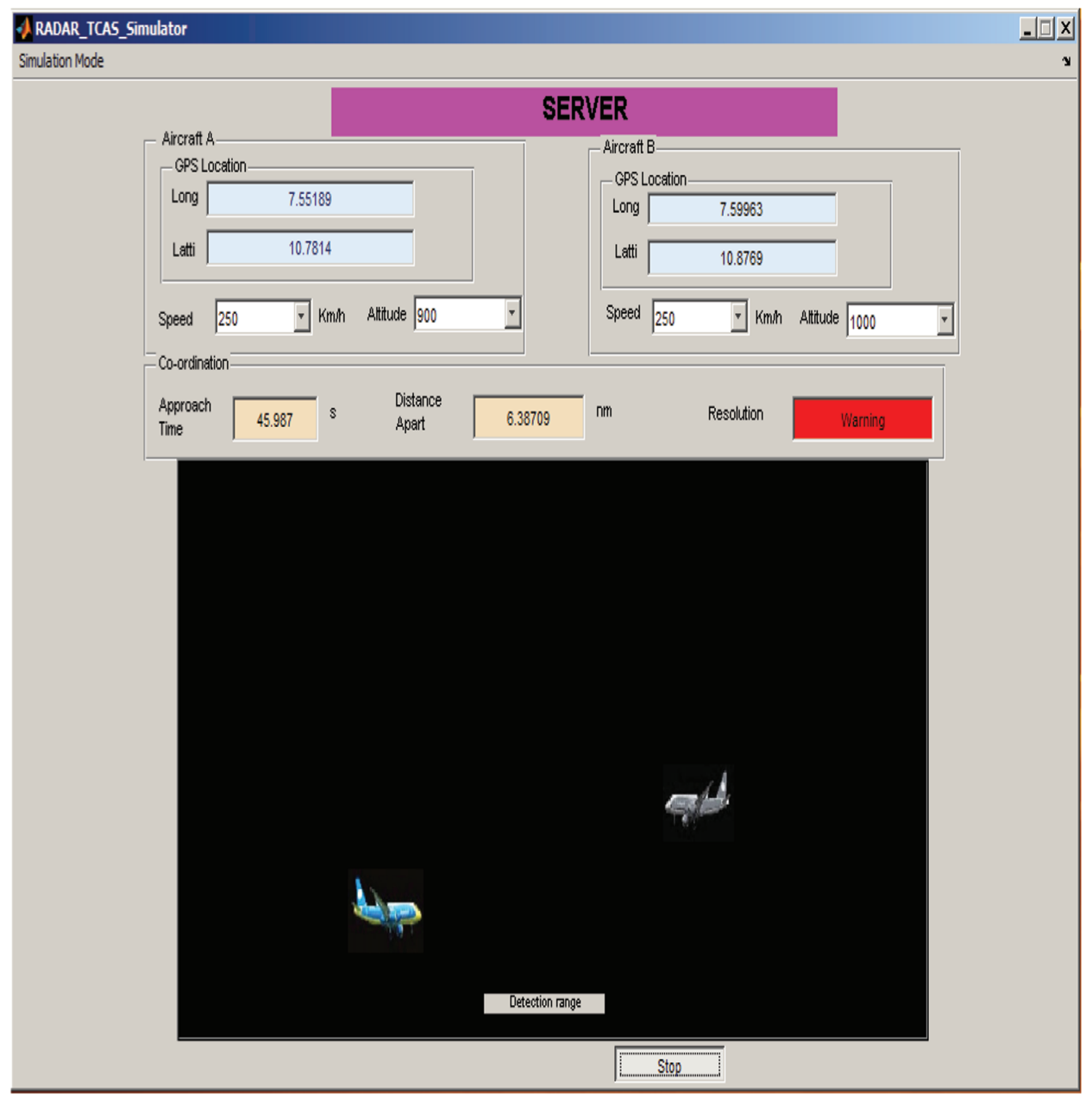

Figure 3. MATLAB display during simulation.

\section{Simulation Results}

In the simulation, after the aircraft were detected and identified, they were placed under surveillance and continuously tracked/interrogated in order to ascertain the required parameters. The radar system gave altitude warnings, while TCAS gave preventive traffic advisories (TA) within 43 seconds. Corrective lateral resolution advisories (RA) were given within 34 seconds when the intruder range was considered dangerous at the closet point of approach (CPA).

The time delay to carry out separation in the approach area was less using an integrated radar and GPS-based TCAS than using an air traffic controller alone, because the information from the GPS satellite about current time, date, and position was displayed continuously (every second) (Wickens, 1999). The system used these trajectory data to monitor the airspace around an aircraft for other aircraft equipped with a corresponding active system and warn pilots of the presence of those aircraft. The system allowed a reduced traffic separation in the terminal areas and moved part of the responsibility of separation from the controller to the pilot to enhance safety. This does not relieve the need for an air traffic controller, but could help to minimize reaction time during periods of high workload.

\section{Future Work}

Future extensions of this work are possible. For instance, there is a need to develop techniques that combine symbolic representation of timing information with symbolic representation of approach control locations. Also, this work could be extended to allow for the possibility of integration of TCAS and the ground proximity warning system, GPWS.

\section{Conclusion}

Using MATLAB simulations, the radar system was integrated with GPS-based TCAS for approach control separation. Results of the simulation showed that aircraft separation after integration is easier and safer than relying only on air traffic controllers. The system gave altitude warnings between aircraft, and successfully discriminated between preventive traffic advisories and corrective lateral resolution advisory when the intruder range or altitude was 
Table 1

Results of Aircraft Simulation in Different Altitude Modes

\begin{tabular}{|c|c|c|c|c|c|c|c|}
\hline \multicolumn{2}{|c|}{ Aircraft A } & \multicolumn{2}{|c|}{ Aircraft B } & \multirow[b]{2}{*}{ Distance Apart (nm) } & \multirow[b]{2}{*}{ TAU (Second) } & \multirow[b]{2}{*}{ Resolution } & \multirow[b]{2}{*}{ System Performance } \\
\hline Long. (Deg) & Lat. (Deg) & Long. (Deg) & Lat. (Deg) & & & & \\
\hline 7.43 & 10.55 & 7.72 & 11.11 & 37.86 & 567.9 & - & No Traffic \\
\hline 7.44 & 10.55 & 7.72 & 11.11 & 37.65 & 564.81 & - & - \\
\hline 7.44 & 10.56 & 7.71 & 11.1 & 36.01 & 540.13 & - & - \\
\hline 7.55 & 10.78 & 7.6 & 10.88 & 6.59 & 98.9 & - & - \\
\hline 7.55 & 10.78 & 7.6 & 10.88 & 6.39 & 95.81 & - & - \\
\hline 7.55 & 10.78 & 7.6 & 10.87 & 5.98 & 89.64 & - & - \\
\hline 7.55 & 10.79 & 7.6 & 10.87 & 5.56 & 83.47 & - & - \\
\hline 7.56 & 10.79 & 7.6 & 10.87 & 5.36 & 80.38 & - & - \\
\hline 7.56 & 10.79 & 7.6 & 10.87 & 5.15 & 77.3 & - & - \\
\hline 7.56 & 10.79 & 7.59 & 10.87 & 4.95 & 74.21 & - & - \\
\hline 7.56 & 10.79 & 7.59 & 10.86 & 4.74 & 71.13 & - & - \\
\hline 7.56 & 10.8 & 7.59 & 10.86 & 4.54 & 68.04 & - & Detection \\
\hline 7.56 & 10.8 & 7.59 & 10.86 & 4.33 & 64.96 & - & - \\
\hline 7.56 & 10.8 & 7.59 & 10.86 & 4.12 & 61.87 & - & - \\
\hline 7.56 & 10.8 & 7.59 & 10.86 & 3.92 & 58.78 & - & - \\
\hline 7.56 & 10.8 & 7.59 & 10.86 & 3.51 & 52.61 & - & - \\
\hline 7.56 & 10.8 & 7.59 & 10.85 & 3.3 & 49.53 & - & - \\
\hline 7.56 & 10.81 & 7.59 & 10.85 & 3.1 & 46.44 & TA & Traffic \\
\hline 7.56 & 10.81 & 7.59 & 10.85 & 2.89 & 43.36 & TA & Traffic \\
\hline 7.57 & 10.81 & 7.59 & 10.85 & 2.68 & 40.27 & TA & Traffic \\
\hline 7.57 & 10.81 & 7.59 & 10.85 & 2.48 & 37.19 & - & - \\
\hline 7.57 & 10.81 & 7.58 & 10.85 & 2.27 & 34.1 & RA & Collision \\
\hline 7.57 & 10.81 & 7.58 & 10.84 & 2.07 & 31.02 & RA & Collision \\
\hline 7.57 & 10.82 & 7.58 & 10.84 & 1.86 & 27.93 & RA & Reverse \\
\hline 7.57 & 10.82 & 7.58 & 10.84 & 1.66 & 24.84 & - & - \\
\hline 7.57 & 10.82 & 7.58 & 10.84 & 1.45 & 21.76 & - & - \\
\hline 7.57 & 10.82 & 7.58 & 10.84 & 1.24 & 18.67 & - & - \\
\hline 7.57 & 10.82 & 7.58 & 10.84 & 1.04 & 15.59 & - & - \\
\hline 7.57 & 10.82 & 7.58 & 10.84 & 0.83 & 12.5 & - & - \\
\hline 7.57 & 10.82 & 7.58 & 10.83 & 0.63 & 9.42 & - & - \\
\hline 7.57 & 10.83 & 7.58 & 10.83 & 0.42 & 6.33 & - & - \\
\hline 7.57 & 10.83 & 7.58 & 10.83 & 0.22 & 3.25 & - & No Traffic \\
\hline 7.58 & 10.83 & 7.58 & 10.83 & 0.01 & 0.16 & - & No Traffic \\
\hline 7.58 & 10.83 & 7.58 & 10.83 & 0.2 & 2.93 & - & No Traffic \\
\hline
\end{tabular}

considered dangerous. It should be noted, however, that the service of an air traffic controller is still required for successful landings, and especially for aerodrome control.

\section{References}

Airbus. (n.d). Required navigational performance. Retrieved from http:// www.airbus.com/innovation/proven-concepts/in-operations/requirednavigation-performance/.

Bakare, K. A., \& Sahalu, J. (2011, August). Modeling and analysis of GPS-based traffic alert and collision avoidance system (TCAS) using "UPPAAL." International Journal of Engineering Science and Technology, 3(8), 6751-6765.

Dennis, G. D. (2003). TSAFE: Building a trusted computing base for air traffic control software (Unpublished master's thesis). Massachusetts Institute of Technology, Cambridge, MS. Retrieved from http://alloy. mit.edu/community/files/gdennis_masters.pdf.

International Civil Aviation Organization. (2002). Surveillance radar and collision avoidance systems (Annex 10, Volume IV). Montreal, Quebec: International Civil Aviation Organization.

Kebabjian, R. (2012). Accident statistics. Retrieved from http://www. planecrashinfo.com/cause.htm.
Lufthansa Airlines. (2005). Lufthansa resource technical training manual (Part 66). Deutz, Cologne: Lufthansa Airlines.

Obiniyi, A. A., \& Bakare, K. A. (2009). Fuzzy logic model for air traffic control system. Paper presented at the $9^{\text {th }}$ Annual International Conference Proceedings for Nigerian Computer Society, Owerri, Nigeria.

Plaettner-Hochwarth, J. K., Zhao, Y. J., \& Robinson, J. E. (2000). A modularized approach for comprehensive air traffic system simulation (AIAA-2000-4478). Reston, VA: American Institute of Aeronautics and Astronautics. Retrieved from http://www.aem.umn. edu/research/atc/download/AIAA-2000-4478.pdf.

Sood, N. \& Wieland, F. (2003).Total airport and airspace model (TAAM) parallelization combining sequential and parallel algorithms for performance enhancement. In S. Chick, P. J. Sanchez, D. Ferrin, \& D. J. Morrice (Eds.), Proceedings of the 2003 Winter Simulation Conference (pp. 1650-1655). Retrieved from http://www.informs-sim. org/wsc03papers/210.pdf.

Wickens, C. (1999). Automation in air traffic control: The human performance issues. In M. W. ScerboM. Mouloua (Eds), Automation technology and Human Performance (pp. 2-10). Mahwah, NJ: Lawrence Erlbaum.

Wolff, C. (2012). Radar tutorial. Retrieved from http://www.radartutorial.eu.

Duncan Aviation. (2013). The NextGen initiative. Retrieved from www. duncanaviation.aero/straighttalk/adsb/nextgen.php. 